Tropical Journal of Pharmaceutical Research May 2019; 18 (5): 1109-1117

ISSN: $1596-5996$ (print); 1596-9827 (electronic)

(C) Pharmacotherapy Group, Faculty of Pharmacy, University of Benin, Benin City, 300001 Nigeria.

\title{
Biological screening and docking studies of unique hybrids synthesized by conventional versus microwave- assisted techniques
}

\author{
NA Virk ${ }^{1}$, Aziz-ur-Rehman ${ }^{1 *}$, MA Abbasi ${ }^{1}$, SZ Siddiqui ${ }^{1}$, A Ashraf ${ }^{2}$, J lqbal ${ }^{3}$, S \\ Rasool $^{1}$, H Khalid ${ }^{4}$, SJ Laulloo ${ }^{5}$, SU Khan ${ }^{6}$, SAA Shah ${ }^{7,8}$ \\ ${ }^{1}$ Department of Chemistry, Government College University, Lahore-54000, ${ }^{2}$ Department of Zoology, Government College \\ University Faisalabad-38040, ${ }^{3}$ Department of Chemistry, University of Lahore-54600, ${ }^{4}$ Department of Chemistry, Forman \\ Christian College University, Lahore-54600, Pakistan, ${ }^{5}$ Department of Chemistry, University of Mauritius, Reduit, Mauritius, \\ ${ }^{6}$ School of Pharmacy, Monash University Malaysia, Jalan Lagoon Selatan, Bandar Sunway, 47500 Subang Jaya, Selangor, \\ ${ }^{7}$ Faculty of Pharmacy; ${ }^{8}$ Atta-ur-Rahman Institute for Natural Products Discovery (AuRIns), Universiti Teknologi MARA, Puncak \\ Alam Campus, 42300 Bandar Puncak Alam, Selangor Darul Ehsan, Malaysia
}

*For correspondence: Email: azizryk@yahoo.com, asmabinm@gmail.com; Tel: (+92)-42-111000010 ext 450

Sent for review: 3 October 2018

Revised accepted: 14 April 2019

\begin{abstract}
Purpose: To carry out the synthesis of various hybrids of 1,2,4-triazole in search of potential therapeutic enzyme inhibitory agents, and carry out docking and bovine serum albumin (BSA) binding studies on docking and bovine serum albumin (BSA) binding studies on the hybrids.

Methods: The target compounds were synthesized by following a multistep protocol. Compound 1 was synthesized from 4-methoxybenzenesulfonyl chloride (a) and ethyl isonipecotate (b). Compound 1 was refluxed with hydrazine to synthesize compound 2, which was converted to compound 3 through two consecutive steps. Compound 4 and different amines (5a-5i), were utilized to synthesize an array of electrophiles (6a-6i). A series of 1,2,4-triazole hybrids (7a-7i) were synthesized at room temperature by stirring together 3 and $6 a-6 i$. The final structures of $7 \mathrm{a}$-7i were elucidated through ${ }^{1} \mathrm{H}$-NMR, ${ }^{13} \mathrm{C}-\mathrm{NMR}$ and El-MS spectroscopy. The BSA binding studies were performed by fluorometric titration. Furthermore, antioxidant and enzyme inhibition activities were determined colorimetrically.

Results: Compound $7 d$ was the most active antioxidant agent, compared to butylated hydroxyanisole (BHA), while compounds $7 d, 7 e, 7 f, 7 g$ and $7 i$ proved to be potent urease inhibitors with half-maximal inhibitory concentration $\left(I C_{50}\right)$ values of $19.5 \pm 0.12,21.1 \pm 0.68,18.2 \pm 0.78,19.9 \pm 0.77$ and $17.9 \pm$ $0.10 \mu \mathrm{M}$, respectively, compared to thiourea with an $I_{50}$ of $24.3 \pm 0.24 \mu \mathrm{M}$. Compounds $7 \mathrm{a}, 7 \mathrm{~b}, 7 \mathrm{~d}$, and $7 e$ exhibited high butyrylcholinesterase inhibition potential, compared to eserine.

Conclusion: The synthesized compounds require studies further as potential therapeutic enzyme inhibitory agents in view of their urease inhibition as well as antioxidant activity.
\end{abstract}

Keywords: Ethyl isonipecotate, 1,2,4-Triazole, Urease inhibition, Butyrylcholinesterase inhibition

This is an Open Access article that uses a fund-ing model which does not charge readers or their institutions for access and distributed under the terms of the Creative Commons Attribution License (http://creativecommons.org/licenses/by/4.0) and the Budapest Open Access Initiative (http://www.budapestopenaccessinitiative.org/read), which permit unrestricted use, distribution, and reproduction in any medium, provided the original work is properly credited.

Tropical Journal of Pharmaceutical Research is indexed by Science Citation Index (SciSearch), Scopus, International Pharmaceutical Abstract, Chemical Abstracts, Embase, Index Copernicus, EBSCO, African Index Medicus, JournalSeek, Journal Citation Reports/Science Edition, Directory of Open Access Journals (DOAJ), African Journal Online, Bioline International, Open-J-Gate and Pharmacy Abstracts

\section{INTRODUCTION}

Nitrogen-based heterocyclic compounds are pharmacologically active agents [1]. These moieties include piperidine [2-5] and 1,2,4triazole [4,6-9], which show different biological activities. On the basis of their biological potential, we designed the current study to 
combine piperidine and 1,2,4-triazole in a single compound to explore their biological potential. Both conventional and microwave-assisted synthetic protocols were utilized. Microwaveassisted synthesis is found to be more suitable in term of purity, time of synthesis and yield [10]. The designed series of compounds were screened for antioxidant and enzyme-inhibitory activity [13], followed by docking studies [11,12]. The docking studies helped elucidate the interaction of the most active synthesized compounds with a specific protein. The BSA binding studies provided more information about the protein interaction of the synthesized compounds.

\section{EXPERIMENTAL}

\section{General}

The ${ }^{1} \mathrm{H}$ - NMR and ${ }^{13} \mathrm{C}$ - NMR spectra were obtained with a Bruker spectrometer operating at 600 and $150 \mathrm{MHz}$, respectively. A Jasco - 320 spectrophotometer was used to collect the IR spectra. Thin layer chromatography helped to determine the completion of reactions and purity of the synthesized compounds. Both Alfa Aeser and Sigma Aldrich provided analytical grade chemicals.

\section{Synthesis of ethyl 1-[(4-methoxyphenyl) sulfonyl]-4-piperidinecarboxylate (1)}

Compound 1 was synthesized by stirring together 4-methoxybenzenesulfonyl chloride (a: $0.04 \mathrm{~mol}$ ) and ethyl isonipecotate (b: $0.04 \mathrm{~mol}$ ) in aqueous medium, maintained at $\mathrm{pH} 9-10$ with $15 \% \mathrm{Na}_{2} \mathrm{CO}_{3}$, for $4 \mathrm{~h}$ at room temperature. Chilled distilled water was then added, and the resulting precipitate was filtered out, washed with distilled water and dried at room temperature.

\section{Synthesis of 1-[(4-methoxyphenyl)sulfonyl] piperidine-4-carbohydrazide (2)}

Compound 2 was synthesized by refluxing an equimolar mixture of compound $\mathbf{1}$ and hydrazine hydrate for $3.5 \mathrm{~h}$ in $\mathrm{EtOH} .2$ was precipitated by the addition of chilled distilled water, filtered out, washed with distilled water and dried at room temperature.

Synthesis of 5-(1-(4-methoxyphenylsulfonyl) piperidin-4-yl)-4-phenyl-4H-1,2,4-triazole-3thiol (3)

Equimolar amounts $(0.5 \mathrm{~mol})$ of compound 2 and phenyl isothiocyanate were refluxed for $3 \mathrm{~h}$ in $\mathrm{EtOH}$ to form an un-cyclized intermediate. The intermediate was refluxed with equimolar $\mathrm{KOH}$ for another $3 \mathrm{~h}$ to obtain the title compound. Compound 3 was precipitated at $\mathrm{pH} 4-5$ by the addition of dil. $\mathrm{HCl}$ to the reaction mixture. The precipitate was filtered out, washed with distilled water and dried at room temperature.

\section{Synthesis of $\mathrm{N}$-(substituted)-2-bromoacetami- $\operatorname{des}(6 a-6 i)$}

Equimolar aryl amines (5a-5i) were stirred with 2bromoacetyl bromide (4) for $2 \mathrm{~h}$ in distilled water at $\mathrm{pH} 9-10$, maintained with $15 \% \mathrm{Na}_{2} \mathrm{CO}_{3}$. The precipitate was filtered out, washed with distilled water and dried at room temperature.

General procedure for synthesis of $\mathrm{N}$ (substituted)-2-[(5-\{1-[(4-methoxyphenyl) sulfonyl]-4-piperidinyl\}-4-phenyl-4H-1,2,4triazol-3-yl)sulfanyl]acetamide (7a-7i)

Conventional method: Compound $\mathbf{3}$ was stirred with an equimolar amount of $\mathrm{NaH}$ in DMF for 30 min. Equimolar electrophile (6a-6i) was added and the reaction mixture stirred for $12-20 \mathrm{~h}$. The precipitate of $\mathbf{7 a - 7 i}$ appeared upon addition of chilled distilled water and was filtered out, washed with distilled water and dried at room temperature.

Microwave-assisted method: Compound $\mathbf{3}$ was stirred with an equimolar amount of $\mathrm{NaH}$ as a catalyst in DMF for $5 \mathrm{~s}$. Equimolar electrophile (6a-6i) was added and the reaction mixture stirred for $34-73 \mathrm{~s}$. The precipitate of $7 \mathbf{a}-7 \mathbf{i}$ appeared upon addition of chilled distilled water and was filtered out, washed with distilled water and dried at room temperature.

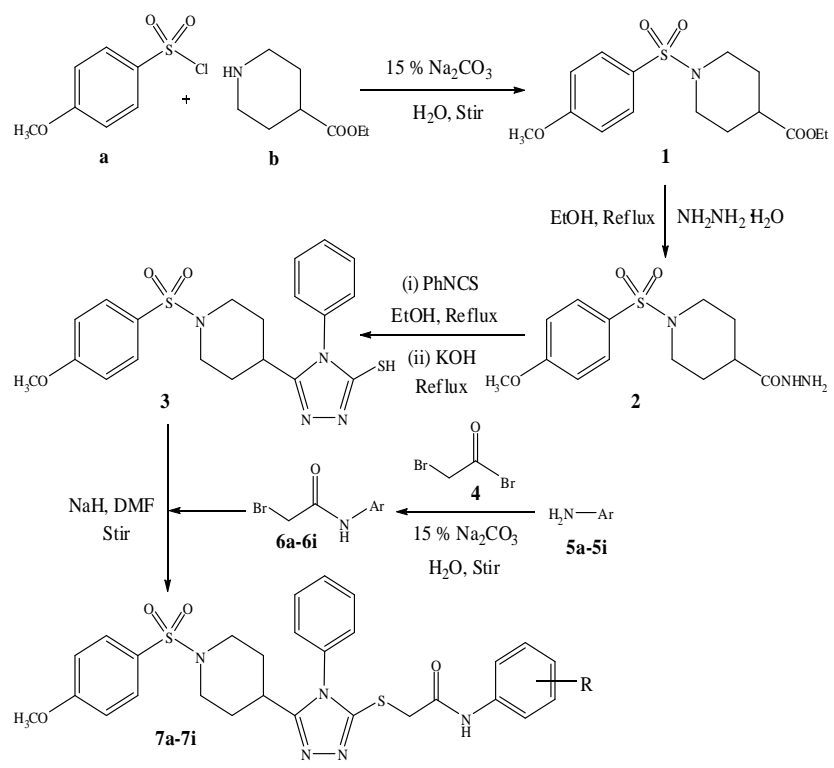

Scheme 1: Synthesis of heterocyclic acetamide derivatives of 1,2,4-triazole. 


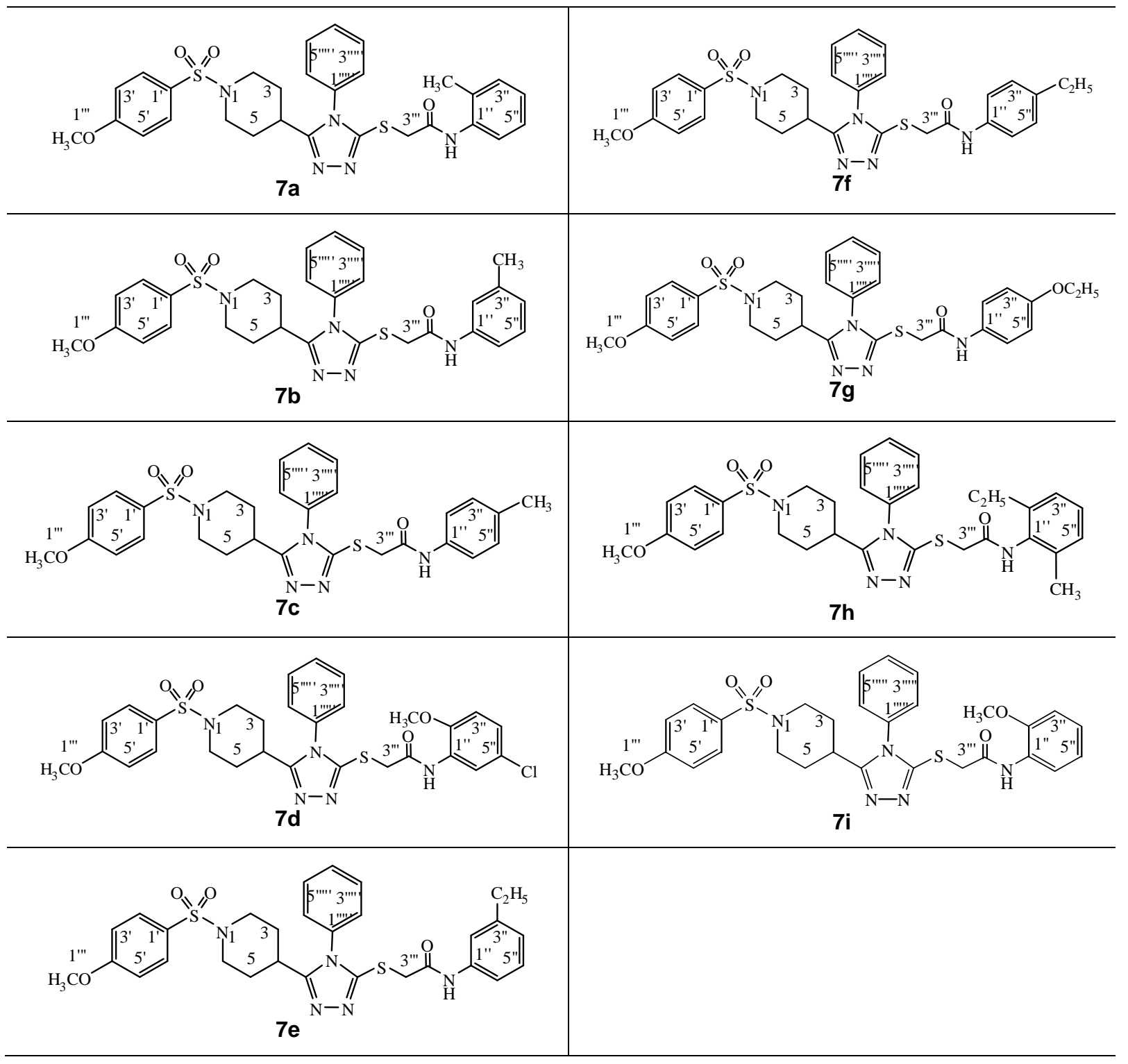

\section{Antioxidant assay (DPPH assay)}

The protocol described by Koleva and coworkers was used with some modifications to measure antioxidant activity [14]. Butylated hydroxyanisole (BHA) was the reference standard. The change in absorbance in the presence of test compound was noted and compared to that of control.

\section{Butyrylcholinesterase (BChE) inhibition assay}

The protocol described by Ellman et al was used with modifications to measure butyrylcholinesterase (BChE) inhibitory activity [15]. Eserine was the reference standard. The change in absorbance in the presence of test compound was noted and compared to that of control.

\section{Urease inhibition assay}

Mobley and coworkers reported a method to measure urease inhibition activity [16] which we used with some modifications. Thiourea was used as the reference standard. The change in absorbance in the presence of test compound was noted and compared to that of control.

\section{Docking studies}

The urease protein structure was obtained from the Protein Data Bank (PDB) ID: 3LA4 with a resolution of $2.05 \AA$ [17]. The calculations of docked molecules were obtained using OpenEye Scientific Software. The different conformations were obtained through OMEGA 3.0.0 (OMEGA, version 2.4.6, 2013), and the binding interactions 
were visualized using Discovery Studio Client v16.1.0 (BIOVIA, 2017).

\section{BSA binding studies}

BSA binding interactions were determined using the method used by Chmara and coworkers [18], with some modifications. Buffer and BSA were purchased from Sigma-Aldrich. Fluorescence was measured with an LS 55 Perkin Elmer fluorescence spectrophotometer [19]. Fluorescence of BSA in the presence of synthesized compounds was recorded and compared with that of BSA alone.

\section{Statistical analysis}

Each experiment was performed in triplicate, and the results are presented as mean \pm SEM. MS Excel 2010 was used to perform statistical analysis with $85 \% \mathrm{Cl}$. The results of enzyme inhibition assays are expressed as $I_{50}$ (concentration for $50 \%$ inhibition) using EZ-Fitz software (Perrella Scientific Inc, USA).

\section{RESULTS}

Hybrids bearing 1,2,4-triazole and piperidine were synthesized according to Scheme 1 . The complete structures of the synthesized compounds are listed in Table 1. Comparison of conventional and microwave-assisted methods is given in Table 2 with regard to time and yield.

\section{Spectral characteristics of synthesized molecules (7a-7i)}

\section{2-[(5-\{1-[(4-Methoxyphenyl)sulfonyl]-4- piperidinyl\}-4-phenyl-4H-1,2,4-triazol-3- yl)sulfanyl]-N-(2-methylphenyl)acetamide (7a)}

White amorphous solid; M.P: $108.0{ }^{\circ} \mathrm{C}$; M.F.: $\mathrm{C}_{29} \mathrm{H}_{31} \mathrm{~N}_{5} \mathrm{O}_{4} \mathrm{~S}_{2}$; M.M.: $577.71 \mathrm{~g} / \mathrm{mol}$; IR $(\mathrm{KBr}, \mathrm{cm}$ $\left.{ }^{1}\right): 2850,1700,1600,1500,1300,1250,1150$, 750; ${ }^{1} \mathrm{H}-\mathrm{NMR}\left(\mathrm{CDCl}_{3}, 600 \mathrm{MHz}, \delta(\mathrm{ppm})\right): 9.88$ ( s, $1 \mathrm{H}, \mathrm{NH}), 7.98\left(\mathrm{~d}, J=10.8 \mathrm{~Hz}, \mathrm{H}-6^{\prime \prime \prime \prime \prime)}\right), 7.58(\mathrm{~d}$, $\left.J=9.4 \mathrm{~Hz}, 2 \mathrm{H}, \mathrm{H}-2^{\prime}, \mathrm{H}-6^{\prime}\right), 7.56-7.55(\mathrm{~m}, 3 \mathrm{H}, \mathrm{H}-$ 3", H-4", H-5"), 7.26-7.21 (m, 4H, H-2", H-6", H4"'"', H-5"'"'), 7.20-7.19 (m, 1H, H-3"'"'), 6.99 (d, J = $\left.9.7 \mathrm{~Hz}, 2 \mathrm{H}, \mathrm{H}-3^{\prime}, \mathrm{H}-5^{\prime}\right), 3.97$ (s, 2H, H-2'"), 3.88 (s, 3H, H-1"'), 3.74-3.71 (m, 2H, $\left.\mathrm{H}_{\mathrm{e}}-2, \mathrm{H}_{\mathrm{e}}-6\right)$, 2.41-2.38 (m, $1 \mathrm{H}, \mathrm{H}-4), 2.35-2.33\left(\mathrm{~m}, 2 \mathrm{H}, \mathrm{H}_{\mathrm{a}}-2\right.$, $\left.\mathrm{H}_{\mathrm{a}}-6\right)$, 2.31 (s, 3H, H-3'"'), 2.03-2.00 (m, $2 \mathrm{H}, \mathrm{H}_{\mathrm{e}}-3$, $\left.\mathrm{H}_{\mathrm{e}}-5\right), 1.88-1.86\left(\mathrm{~m}, 2 \mathrm{H}, \mathrm{H}_{\mathrm{a}}-3, \mathrm{H}_{\mathrm{a}}-5\right) ;{ }^{13} \mathrm{C}-\mathrm{NMR}$ $\left(\mathrm{CDCl}_{3}, 150 \mathrm{MHz}, \delta(\mathrm{ppm})\right): 165.58$ (C-4'), 163.22 (C-5"'"), 157.90 (C-3"'"), 151.88 (C-6"'"'), 136.22 (C-1"'"), 131.39 (C-1"), 130.75 (C-4"), 130.43 (C-3", C-5"), 129.64 (C-2", C-6"), 128.74 (C-2', C-6'), 127.45 (C-1'), 126.11 (C-2"'"'), 125.78 (C-6"'"'), 125.22 (C-3"'"'), 125.11 (C-5"'"'),
124.21 (C-4"'"'), 114.20 (C-3', C-5'), 55.66 (C-1"'), 45.49 (C-2, C-6), 36.34 (C-2"'), 32.01 (C-4), 29.30 (C-3, C-5 ), 17.43 (C-3"'); EIMS (m/z): 577 $[\mathrm{M}]^{+}, 371,295,255,171,108,106,52$.

\section{2-[(5-\{1-[(4-Methoxyphenyl)sulfonyl]-4- piperidinyl\}-4-phenyl-4H-1,2,4-triazol-3- yl)sulfanyl]-N-(3-methylphenyl)acetamide (7b)}

White amorphous solid; M.P: $105.3{ }^{\circ} \mathrm{C}$; M.F.: $\mathrm{C}_{29} \mathrm{H}_{31} \mathrm{~N}_{5} \mathrm{O}_{4} \mathrm{~S}_{2}$; M.M.: $577.71 \mathrm{~g} / \mathrm{mol}$; IR $\left(\mathrm{KBr}, \mathrm{cm}^{-}\right.$ $\left.{ }^{1}\right)$ : 2850, 1700, 1600, 1500, 1325, 1250, 1100, 750; ${ }^{1} \mathrm{H}-\mathrm{NMR}\left(\mathrm{CDCl}_{3}, 600 \mathrm{MHz}, \delta(\mathrm{ppm})\right): 10.14$ (s, $1 \mathrm{H}, \mathrm{NH}), 7.69\left(\mathrm{~d}, J=8.7 \mathrm{~Hz}, 2 \mathrm{H}, \mathrm{H}-2^{\prime}, \mathrm{H}-6^{\prime}\right)$, 7.63-7.55 (m, 3H, H-3", H-4", H-5"), 7.45 (s, 1H,

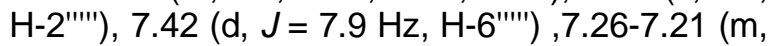
$\left.3 \mathrm{H}, \mathrm{H}-2 ", \mathrm{H}-6 ", \mathrm{H}-5^{\prime \prime \prime \prime)}\right), 6.99$ (d, J = $8.7 \mathrm{~Hz}, 2 \mathrm{H}$, H-3', H-5'), 6.94 (d, J = $7.3 \mathrm{~Hz}, 1 \mathrm{H}, \mathrm{H}-4{ }^{\prime \prime \prime \prime)}, 3.90$ (s, 2H, H- 2"'), 3.88 (s, 3H, H-1"'), 3.78-3.74 (m, $\left.2 \mathrm{H}, \mathrm{H}_{\mathrm{e}}-2, \mathrm{H}_{\mathrm{e}}-6\right), 2.52-2.49(\mathrm{~m}, 1 \mathrm{H}, \mathrm{H}-4), 2.36(\mathrm{~s}$, $\left.3 \mathrm{H}, \mathrm{H}-3^{\prime \prime \prime}\right), 2.34-2.28\left(\mathrm{~m}, 2 \mathrm{H}, \mathrm{H}_{\mathrm{a}}-2, \mathrm{H}_{\mathrm{a}}-6\right)$, 2.06$2.00\left(\mathrm{~m}, 2 \mathrm{H}, \mathrm{H}_{\mathrm{e}}-3, \mathrm{H}_{\mathrm{e}}-5\right), 1.90-1.86\left(\mathrm{~m}, 2 \mathrm{H}, \mathrm{H}_{\mathrm{a}}-3\right.$, $\left.\mathrm{H}_{\mathrm{a}}-5\right) ;{ }^{13} \mathrm{C}-\mathrm{NMR}\left(\mathrm{CDCl}_{3}, 150 \mathrm{MHz}, \delta\right.$ (ppm)): 166.54 (C-4'), 163.00 (C-5"'"), 157.96 (C-3"'"'), 154.88 (C-6"'"'), 138.82 (C-1"'"'), 138.37 (C-1"), 132.40 (C-3"'"'), 130.79 (C-6"'"'), 130.44 (C-3", C5"), 130.19 (C-4"), 129.77 (C-2", C-6"), 128.72 (C-2"'"'), 126.96 (C-2', C-6'), 125.90 (C-1'), 120.36 (C-5"'"'), 116.93 (C-4"'"'), 114.21 (C-3', C5'), 55.60 (C-1'"), 45.45 (C-2, C-6), 36.11 (C-2"'), 32.08 (C-4), 29.49 (C-3, C-5), 21.47 (C-3"'); EIMS (m/z): $577[\mathrm{M}]^{+}, 371,295,255,171,108$, 106, 66.

\section{2-[(5-\{1-[(4-Methoxyphenyl)sulfonyl]-4- piperidinyl\}-4-phenyl-4H-1,2,4-triazol-3- yl)sulfanyl]-N-(4-methylphenyl)acetamide (7c)}

White amorphous solid; M.P: $114.7{ }^{\circ} \mathrm{C}$; M.F.: $\mathrm{C}_{29} \mathrm{H}_{31} \mathrm{~N}_{5} \mathrm{O}_{4} \mathrm{~S}_{2}$; M.M.: $577.71 \mathrm{~g} / \mathrm{mol}$; IR $\left(\mathrm{KBr}, \mathrm{cm}^{-}\right.$ $\left.{ }^{1}\right): 2850,1700,1600,1525,1350,1250,1100$, 725; ${ }^{1} \mathrm{H}-\mathrm{NMR}\left(\mathrm{CDCl}_{3}, 600 \mathrm{MHz}, \delta\right.$ (ppm)): 10.12 (s, $1 \mathrm{H}, \mathrm{NH}), 7.69\left(\mathrm{~d}, J=8.8 \mathrm{~Hz}, 2 \mathrm{H}, \mathrm{H}-2^{\prime}, \mathrm{H}-6^{\prime}\right)$, 7.59-7.55 (m, 2H, H-3", H-4"), 7.50 (d, J = 8.2 $\mathrm{Hz}, 2 \mathrm{H}, \mathrm{H}-2$ '"', $\mathrm{H}-6^{\prime \prime \prime \prime '), ~} 7.23$ (d, J = 6.7 Hz, 2H, H2", H-6"), 7.14 (d, J = 8.1 Hz, 2H, H-3"'"', H-5"'"'), 6.99 (d, J = 8.8 Hz, 2H, H-3', H-5'), 3.88 (s, $5 \mathrm{H}$, H-1"', H-2"'), 3.77- $3.75\left(\mathrm{~m}, 2 \mathrm{H}, \mathrm{H}_{\mathrm{e}}-2, \mathrm{H}_{\mathrm{e}}-6\right)$, 2.52$2.49(\mathrm{~m}, 1 \mathrm{H}, \mathrm{H}-4), 2.31-2.28\left(\mathrm{~m}, 2 \mathrm{H}, \mathrm{H}_{\mathrm{a}}-2, \mathrm{H}_{\mathrm{a}}-6\right)$, 2.06-2.00 (m, $\left.2 \mathrm{H}, \mathrm{H}_{\mathrm{e}}-3, \mathrm{H}_{\mathrm{e}}-5\right)$, 1.88-1.85 (m, $2 \mathrm{H}$, $\left.\mathrm{H}_{\mathrm{a}}-3, \mathrm{H}_{\mathrm{a}}-5\right), 1.71\left(\mathrm{~s}, 3 \mathrm{H}, \mathrm{H}-3^{\prime \prime \prime}\right) ;{ }^{13} \mathrm{C}-\mathrm{NMR}\left(\mathrm{CDCl}_{3}\right.$, $150 \mathrm{MHz}, \delta$ (ppm)): 166.39 (C-4'), 163.00 (C-5"'"), 157.95 (C-3"'"), 152.88 (C-6"'"'), 135.66 (C-1"'"'), 133.81 (C-1"), 132.40 (C-4"'"), 130.78 (C-4"), 130.43 (C-3", C-5"), 129.77 (C-2", C-6"), 129.37 (C-3"'"', 5"'"'), 127.80 (C-1'), 126.96 (C-2', C-6'), 119.79 (C-6"'"', C-2"'"'), 114.21 (C-3', C-5'), 55.60 (C-1"'), 45.49 (C-2, C-6), 36.05 (C-2"'), 32.04 (C4), 29.40 (C-3, C-5), 20.88 (C-3"'); EIMS ( $\mathrm{m} / \mathrm{z})$ : $577[\mathrm{M}]^{+}, 371,295,255,171,108,106,66$. 
$\mathrm{N}$-(5-Chloro-2-methoxyphenyl)-2-[(5-\{1-[(4methoxyphenyl)sulfonyl]-4-piperidinyl\}-4phenyl-4H-1,2,4-triazol-3yl)sulfanyl]acetamide (7d)

Light-pink amorphous solid; M.P: $205.3^{\circ} \mathrm{C}$; M.F.: $\mathrm{C}_{29} \mathrm{H}_{30} \mathrm{~N}_{5} \mathrm{O}_{4} \mathrm{~S}_{2}$; M.M.: $628.16 \mathrm{~g} / \mathrm{mol}$; IR $\left(\mathrm{KBr}, \mathrm{cm}^{-}\right.$ $\left.{ }^{1}\right): 2850,1700,1600,1550,1350,1250,1150$, 725; ${ }^{1} \mathrm{H}-\mathrm{NMR}\left(\mathrm{CDCl}_{3}, 600 \mathrm{MHz}, \delta\right.$ (ppm)): 10.22 (s, $1 \mathrm{H}, \mathrm{NH}), 8.42\left(\mathrm{~s}, 1 \mathrm{H}, \mathrm{H}-6 \mathrm{6}^{\prime \prime \prime \prime}\right), 7.69(\mathrm{~d}, J=8.1$ $\left.\mathrm{Hz}, 2 \mathrm{H}, \mathrm{H}-2^{\prime}, \mathrm{H}-6^{\prime}\right), 7.59-7.54$ (m, 3H, H-3", H-4", $\mathrm{H}-5 "), 7.24$ (d, $J=7.0 \mathrm{~Hz}, 2 \mathrm{H}, \mathrm{H}-2 ", \mathrm{H}-6 "), 7.01$ (d, $\left.J=8.7 \mathrm{~Hz}, 1 \mathrm{H}, \mathrm{H}-3^{\prime \prime \prime \prime}\right), 6.99(\mathrm{~d}, J=8.0 \mathrm{~Hz}$, $\left.2 \mathrm{H}, \mathrm{H}-3^{\prime}, \mathrm{H}-5^{\prime}\right), 6.78$ (d, J = 8.6 Hz, 1H, H-4"'"'), 3.98 (s, 2H, H- 2"'), 3.90 (s, 3H, H- 3"'), 3.88 (s, $\left.3 \mathrm{H}, \mathrm{H}-1{ }^{\prime \prime \prime}\right), 3.76-3.74\left(\mathrm{~m}, 2 \mathrm{H}, \mathrm{H}_{\mathrm{e}}-2, \mathrm{H}_{\mathrm{e}}-6\right)$, 2.54$2.51(\mathrm{~m}, 1 \mathrm{H}, \mathrm{H}-4), 2.37-2.33\left(\mathrm{~m}, 2 \mathrm{H}, \mathrm{H}_{\mathrm{a}}-2, \mathrm{H}_{\mathrm{a}}-6\right)$, 2.07-2.01 (m, $\left.2 \mathrm{H}, \mathrm{H}_{\mathrm{e}}-3, \mathrm{H}_{\mathrm{e}}-5\right), 1.89-1.73(\mathrm{~m}, 2 \mathrm{H}$, $\left.\mathrm{H}_{\mathrm{a}}-3, \mathrm{H}_{\mathrm{a}}-5\right) ;{ }^{13} \mathrm{C}-\mathrm{NMR} \quad\left(\mathrm{CDCl}_{3}, 150 \mathrm{MHz}, \delta\right.$ (ppm)): 166.71 (C-4'), 163.00 C-5"'"), 157.94 (C3"'"), 152.02 (C -6"'"), 147.25 (C-2"'"'), 132.46 (C1"''), 130.67 (C-1"), 132.40 (C-4"'"'), 130.41 (C3", C-5"), 129.75 (C-2", C-6"), 128.96 (C-4"), 127.77 (C-5"'"'), 126.92 (C-2', C-6'), 125.76 (C1'), 123.35 (C-6"'"'), 119.91 (C-3"'"'), 114.22 (C-3', C-5'), 110.84 (C-4"'"'), 56.05 (C -3"'), 55.61 (C1"'), 45.45 (C-2, C-6), 35.88 (C-2'"), 31.76 (C-4), 29.51 (C-3, C-5); EIMS (m/z): $628\left[\mathrm{IM}^{+}, 371,295\right.$, 255, 171, 108, 106, 52.

\section{N-(2-Ethylphenyl)-2-[(5-\{1-[(4- methoxyphenyl)sulfonyl]-4-piperidinyl\}-4- phenyl-4H-1,2,4-triazol-3- yl)sulfanyl]acetamide (7e)}

White amorphous solid; M.P: $157.3{ }^{\circ} \mathrm{C}$; M.F.: $\mathrm{C}_{30} \mathrm{H}_{35} \mathrm{~N}_{5} \mathrm{O}_{4} \mathrm{~S}_{2}$; M.M.: $593.76 \mathrm{~g} / \mathrm{mol}$; IR $(\mathrm{KBr}, \mathrm{cm}$ $\left.{ }^{1}\right): 2850,1700,1600,1550,1325,1250,1150$, 720; ${ }^{1} \mathrm{H}-\mathrm{NMR}\left(\mathrm{CDCl}_{3}, 600 \mathrm{MHz}, \delta\right.$ (ppm)): 9.67 (s, $1 \mathrm{H}, \mathrm{NH}), 7.90\left(\mathrm{~d}, J=7.9 \mathrm{~Hz}, 1 \mathrm{H}, \mathrm{H}-6^{\prime \prime \prime \prime ')}, 7.69\right.$ (d, $\left.J=8.7 \mathrm{~Hz}, 2 \mathrm{H}, \mathrm{H}-2^{\prime}, \mathrm{H}-6^{\prime}\right), 7.60-7.55(\mathrm{~m}, 3 \mathrm{H}$, H-3", H-4", H-5"), 7.23-7.20 (m, 3H, H-2", H-6", H-5"'"'), 7.13 (t, $\left.J=7.3 \mathrm{~Hz}, 1 \mathrm{H}, \mathrm{H}-4^{\prime \prime \prime \prime \prime)}\right), 6.99$ (d, $J$ $\left.=8.7 \mathrm{~Hz}, 2 \mathrm{H}, \mathrm{H}-3^{\prime}, \mathrm{H}-5^{\prime}\right), 3.98$ (s, 2H, H-2'"), 3.88 (s, 3H, H- 1"'), 3.73-3.70 (m, 2H, $\left.\mathrm{H}_{\mathrm{e}}-2, \mathrm{H}-6\right), 2.64$ (q, J = 5.4 Hz, 2H, H-3"'), 2.59-2.53( m, 1H, H-4), 2.40-2.37 (m, $\left.2 \mathrm{H}, \mathrm{H}_{\mathrm{a}}-2, \mathrm{H}_{\mathrm{a}}-6\right), 2.03-2.00(\mathrm{~m}, 2 \mathrm{H}$, $\left.\mathrm{H}_{\mathrm{e}}-3, \mathrm{H}_{\mathrm{e}}-5\right), 1.88-1.85\left(\mathrm{~m}, 2 \mathrm{H}, \mathrm{H}_{\mathrm{a}}-3, \mathrm{H}_{\mathrm{a}}-5\right), 1.14$ (t, $\left.J=3.2 \mathrm{~Hz}, 3 \mathrm{H}, \mathrm{H}-4{ }^{\prime \prime \prime}\right) ;{ }^{13} \mathrm{C}-\mathrm{NMR}\left(\mathrm{CDCl}_{3}, 150\right.$ $\mathrm{MHz}, \delta(\mathrm{ppm})): 167.08$ (C-4'), 163.00 (C-5"'"'), 158.08 (C-3"'"'), 152.44 (C-6"'"), 135.38 (C-1"'"'), 135.37 (C-1"), 130.77 (C-4"), 130.46 (C-3", C5"), 129.74 (C-2", C-6"), 128.74 (C-2"'"'), 128.00 (C-1'), 126.91 (C-2', C-6'), 126.40 (C-5"'"'), 125.28 (C-6"'"'), 123.27 (C-4"'"'), 121.55 (C-3"'"'), 114.21 (C-3', C-5'), 55.57 (C-1'"'), 45.30 (C-2, C6), 35.59 (C-2"'), 31.77 (C-4), 29.38 (C-3, C-5), 24.60 (C-3"'), 14.20 (C-4"'); EIMS (m/z): 593 $[\mathrm{M}]^{+}, 371,295,255,171,108,106,66$.

\section{N-(4-Ethylphenyl)-2-[(5-\{1-[(4- methoxyphenyl)sulfonyl]-4-piperidinyl\}-4- phenyl-4H-1,2,4-triazol-3- yl)sulfanyl]acetamide (7f)}

White amorphous solid; M.P: $130.0{ }^{\circ} \mathrm{C}$; M.F.: $\mathrm{C}_{30} \mathrm{H}_{33} \mathrm{~N}_{5} \mathrm{O}_{4} \mathrm{~S}_{2}$; M.M.: $591.74 \mathrm{~g} / \mathrm{mol}$; IR $\left(\mathrm{KBr}, \mathrm{cm}^{-}\right.$ $\left.{ }^{1}\right): 2850,1700,1600,1550,1350,1250,1150$, 750; ${ }^{1} \mathrm{H}-\mathrm{NMR}\left(\mathrm{CDCl}_{3}, 600 \mathrm{MHz}, \delta\right.$ (ppm)): 10.13 (s, $1 \mathrm{H}, \mathrm{NH}), 7.68\left(\mathrm{~d}, J=8.8 \mathrm{~Hz}, 2 \mathrm{H}, \mathrm{H}-2^{\prime}, \mathrm{H}-6^{\prime}\right)$, 7.60-7.55 (m, 3H, H-3", H-4", H-5"), 7.53 (d, J= $8.4 \mathrm{~Hz}, 2 \mathrm{H}, \mathrm{H}-2$ "'"', H-6"'"'), 7.23 (dd, $J=7.8,2.7$ $\mathrm{Hz}, 2 \mathrm{H}, \mathrm{H}-2$ ", H-6"), 7.16 (d, J = 8.3 Hz, 2H, H3"'", $\mathrm{H}-5^{\prime \prime \prime \prime \prime)}, 6.99$ (d, $\left.J=8.8 \mathrm{~Hz}, 2 \mathrm{H}, \mathrm{H}-3^{\prime}, \mathrm{H}-5^{\prime}\right)$, 3.88 (s, 2H, H-2'"), 3.87 (s, 3H, H-1"'), 3.77-3.75 (m, $\left.2 \mathrm{H}, \mathrm{H}-2, \mathrm{H}_{\mathrm{e}}-6\right), 2.63$ (q, J = 3.9 Hz, 2H, H3"'), 2.52-2.49 (m, 1H, H-4), 2.32-2.26 (m, $2 \mathrm{H}$, $\left.\mathrm{H}_{\mathrm{a}}-2, \mathrm{H}_{\mathrm{a}}-6\right), 2.06-2.00\left(\mathrm{~m}, 2 \mathrm{H}, \mathrm{H}_{\mathrm{e}}-3, \mathrm{H}_{\mathrm{e}}-5\right)$, 1.99$1.85\left(\mathrm{~m}, 2 \mathrm{H}, \mathrm{H}_{\mathrm{a}}-3, \mathrm{H}_{\mathrm{a}}-5\right), 1.22(\mathrm{t}, J=3.5 \mathrm{~Hz}, 3 \mathrm{H}$, $\mathrm{H}-4$ "'); ${ }^{13} \mathrm{C}-\mathrm{NMR}\left(\mathrm{CDCl}_{3}, 150 \mathrm{MHz}, \delta\right.$ (ppm)): 166.41 (C-4'), 163.01 (C-5"'"), 157.95 (C-3"'"), 152.85 (C-6"'"), 140.32 (C-1"'"'), 135.84 (C-1"), 132.38 (C-4"'"'), 130.78 (C-4"), 130.44 (C-3", C5"), 129.77 (C-2", C-6"), 128.21 (C-2', C-6'), 127.74 (C-1'), 126.96 (C-3"'"', C-5"'"), 119.87 (C2"'", C-6"'"'), 114.22 (C-3', C-5'), 55.61 (C-1"'), 45.51 (C-2, C-6), 36.02 (C-2"'), 32.02 (C-4), 29.40 (C-3, C-5), 28.34 (C-3"'), 15.75 (C-4"'); EIMS (m/z): $591[\mathrm{M}]^{+}, 371,295,255,171,108$, 106, 52.

\section{N-(4-Ethoxyphenyl)-2-[(5-\{1-[(4- methoxyphenyl)sulfonyl]-4-piperidinyl\}-4- phenyl-4H-1,2,4-triazol-3- yl)sulfanyl]acetamide (7g)}

Light-pink amorphous solid; M.P: $117.0{ }^{\circ} \mathrm{C}$; M.F.: $\mathrm{C}_{30} \mathrm{H}_{33} \mathrm{~N}_{5} \mathrm{O}_{5} \mathrm{~S}_{2}$; M.M.: $607.74 \mathrm{~g} / \mathrm{mol}$; IR $\left(\mathrm{KBr}, \mathrm{cm}^{-}\right.$ $\left.{ }^{1}\right)$ : 2850, 1700, 1600, 1500, 1320, 1250, 1150, 750; ${ }^{1} \mathrm{H}-\mathrm{NMR}\left(\mathrm{CDCl}_{3}, 600 \mathrm{MHz}, \delta\right.$ (ppm)): 10.08 (s, $1 \mathrm{H}, \mathrm{NH}), 7.69$ (d, J = 8.8 Hz, 2H, H-2', H-6'), 7.60-7.55 (m, 3H, H-3", H-4", H-5"), 7.52 (d, J= $\left.9.0 \mathrm{~Hz}, 2 \mathrm{H}, \mathrm{H}-2^{2 ' " ',}, \mathrm{H}-6^{\prime \prime \prime \prime)}\right), 7.23$ (dd, $J=7.8,3.8$ $\mathrm{Hz}, 2 \mathrm{H}, \mathrm{H}-2 ", \mathrm{H}-6 "), 6.99$ (d, J=8.8 Hz, 2H, H-3', $\left.\mathrm{H}-5^{\prime}\right), 6.87$ (d, J = 9.0 Hz, 2H, H-3"'"', H-5"'"'), 4.02 (q, J = 5.1 Hz, 2H, H-3"'), 3.88 (s, 3H, H-1"'), 3.87 (s, 2H, H-2"'), 3.77-3.74 (m, $\left.2 \mathrm{H}, \mathrm{H}_{\mathrm{e}}-2, \mathrm{H}_{\mathrm{e}}-6\right)$, 2.53-2.49 (m, $1 \mathrm{H}, \mathrm{H}-4), 2.32-2.29\left(\mathrm{~m}, 2 \mathrm{H}, \mathrm{H}_{\mathrm{a}}-2\right.$, $\left.\mathrm{H}_{\mathrm{a}}-6\right)$, 2.06-2.00 (m, $\left.2 \mathrm{H}, \mathrm{H}_{\mathrm{e}}-3, \mathrm{H}_{\mathrm{e}}-5\right)$, 1.88-1.85 (m, $\left.2 \mathrm{H}, \mathrm{H}_{\mathrm{a}}-3, \mathrm{H}_{\mathrm{a}}-5\right), 1.41$ (t, J = 2.7 Hz, 3H, H-4"'); ${ }^{13} \mathrm{C}-\mathrm{NMR}\left(\mathrm{CDCl}_{3}, 150 \mathrm{MHz}, \delta(\mathrm{ppm})\right): 166.25$ (C4'), 163.00 (C-5"'), 157.94 (C-3"'"'), 155.65 (C4"'"), 152.92 (C-6"'"), 132.41 (C-1"'"'), 131.33 (C1"), 130.78 (C-4"), 130.43 (C-3", C-5"), 129.77 (C-2", C-6"), 127.77 (C-1'), 126.96 (C-2', C-6'), 121.34 (C-3"'"', C-5"'"'), 114.70 (C-2"'"', C-6"'"'), 114.21 (C-3', C-5'), 63.70 (C-3"'), 55.60 (C-1"'), 45.49 (C-2, C-6), 36.00 (C-2"'), 32.04 (C-4), 
29.40 (C-3, C-5), 14.84 (C-4'"'); EIMS (m/z): 607 $[\mathrm{M}]^{+}, 371,295,255,171,108,106,52$.

\section{N-(2-Ethyl-6-methylphenyl)-2-[(5-\{1-[(4- methoxyphenyl)sulfonyl]-4-piperidinyl\}-4- phenyl-4H-1,2,4-triazol-3- yl)sulfanyl]acetamide (7h)}

White amorphous solid; M.P: $110.0{ }^{\circ} \mathrm{C}$; M.F.: $\mathrm{C}_{31} \mathrm{H}_{35} \mathrm{~N}_{5} \mathrm{O}_{4} \mathrm{~S}_{2}$; M.M.: $605.77 \mathrm{~g} / \mathrm{mol}$; IR (KBr, cm $\left.{ }^{1}\right): 2850,1700,1600,1500,1350,1250,1150$, 750; ${ }^{1} \mathrm{H}-\mathrm{NMR}\left(\mathrm{CDCl}_{3}, 600 \mathrm{MHz}, \delta(\mathrm{ppm})\right): 9.34$ (s, $1 \mathrm{H}, \mathrm{NH}), 7.68\left(\mathrm{~d}, J=8.6 \mathrm{~Hz}, 2 \mathrm{H}, \mathrm{H}-2^{\prime}, \mathrm{H}-6^{\prime}\right)$, 7.63-7.55 (m, 3H, H-3", H-4", H-5"), 7.23 (br. s, 2H, H-2", H-6"), 7.16-7.09 (m, 3H, H-3"'"', H-4"'"', $\left.\mathrm{H}-5^{\prime \prime \prime \prime}\right), 6.98$ (d, $\left.J=8.7 \mathrm{~Hz}, 2 \mathrm{H}, \mathrm{H}-3^{\prime}, \mathrm{H}-5^{\prime}\right), 4.00$ (s, 2H, H-2"'), 3.86 (s, 3H, H-1"'), 3.73-3.70 (m, $\left.2 \mathrm{H}, \mathrm{H}_{\mathrm{e}}-2, \mathrm{H}_{\mathrm{e}}-6\right), 2.53$ (q, $J=3.8 \mathrm{~Hz}, 2 \mathrm{H}, \mathrm{H}-3^{\prime \prime \prime}$ ), 2.39-2.35 (m, 3H, H-4, $\left.\mathrm{H}_{\mathrm{a}}-2, \mathrm{H}_{\mathrm{a}}-6\right), 2.19(\mathrm{~s}, 3 \mathrm{H}$, H-5'"), 2.03-1.97 (m, 2H, $\left.\mathrm{H}_{\mathrm{e}}-3, \mathrm{H}_{\mathrm{e}}-5\right), 1.87-1.85$ $\left(\mathrm{m}, 2 \mathrm{H}, \mathrm{H}_{\mathrm{a}}-3, \mathrm{H}_{\mathrm{a}}-5\right), 1.09$ (t, J = 5.1 Hz, 3H, H-4'"); ${ }^{13} \mathrm{C}-\mathrm{NMR}\left(\mathrm{CDCl}_{3}, 150 \mathrm{MHz}, \delta\right.$ (ppm)): 166.23 (C4'), 163.01 (C-5"'"), 157.99 (C-3"'"), 152.90 (C6"''), 141.06 (C-1"'"'), 133.00 (C-1"), 130.81(C4"), 130.49 (C-3", C-5"), 129.73(C-2", C-6"), 128.18 (C-6"'"'), 127.57 (C-2"'"'), 126.90 (C-2', C$\left.6^{\prime}\right), 126.38$ (C-1'), 123.89 (C-5"'"'), 121.90 (C3"'"'), 118.23 (C-4"'"'), 114.22 (C-3', C-5'), 55.56 (C-1"'), 45.30 (C-2, C-6), 34.81 (C-2"'), 31.87 (C4), 29.36 (C-3, C-5), 24.87 (C-3"'), 18.22 (C-5'"'), 14.62 (C-4"'); EIMS (m/z): 605 [M] $^{+}, 371,295$, $255,171,108,106,66$.

\section{N-(2-Methoxyphenyl)-2-[(5-\{1-[(4- methoxyphenyl)sulfonyl]-4-piperidinyl\}-4- phenyl-4H-1,2,4-triazol-3- yl)sulfanyl]acetamide (7i)}

White amorphous solid; yield: $87 \%$; M.P: 120.0 ${ }^{\circ} \mathrm{C}$; M.F.: $\mathrm{C}_{30} \mathrm{H}_{33} \mathrm{~N}_{5} \mathrm{O}_{5} \mathrm{~S}_{2}$; M.M.: $575.67 \mathrm{~g} / \mathrm{mol}$; IR $\left(\mathrm{KBr}, \mathrm{cm}^{-1}\right): 2825,1700,1600,1500,1320$, 1250, 1150, 750; ${ }^{1} \mathrm{H}-\mathrm{NMR}\left(\mathrm{CDCl}_{3}, 600 \mathrm{MHz}, \delta\right.$ (ppm)): $9.97(\mathrm{~s}, 1 \mathrm{H}, \mathrm{NH}), 8.35(\mathrm{~d}, J=9.0 \mathrm{~Hz}, 1 \mathrm{H}$, H-6"'"'), 7.69 (d, $\left.J=8.8 \mathrm{~Hz}, 2 \mathrm{H}, \mathrm{H}-2^{\prime}, \mathrm{H}-6^{\prime}\right), 7.57-$ 7.54 (m, 3H, H-3", H-4", H-5"), 7.24 (dd, J =7.4, $2.2 \mathrm{~Hz}, 2 \mathrm{H}, \mathrm{H}-2 ", \mathrm{H}-6 "), 7.06$ (t, $J=7.5 \mathrm{~Hz}, 1 \mathrm{H}$, $\mathrm{H}-5^{\prime \prime \prime \prime \prime), ~} 6.98$ (d, $\left.J=8.8 \mathrm{~Hz}, 2 \mathrm{H}, \mathrm{H}-3^{\prime}, \mathrm{H}-5^{\prime}\right)$, $6.94\left(\mathrm{t}, J=7.3 \mathrm{~Hz}, 1 \mathrm{H}, \mathrm{H}-4^{\prime \prime \prime \prime}\right), 6.89(\mathrm{~d}, J=8.1 \mathrm{~Hz}$, $1 \mathrm{H}, \mathrm{H}-3^{\prime \prime \prime \prime \prime)}, 4.01$ (s, 2H, H-2"'), 3.88 (s, 3H, H1"'), 3.76 (s, 3H, H-3"'), 3.74-3.72 (m, $2 \mathrm{H}, \mathrm{H}_{\mathrm{e}}-2$, $\left.\mathrm{H}_{\mathrm{e}}-6\right)$, 2.54-2.52 (m, 1H, H-4), 2.39-2.36 (m, 2H, $\left.\mathrm{H}_{\mathrm{a}}-2, \mathrm{H}_{\mathrm{a}}-6\right), 2.05-2.02\left(\mathrm{~m}, 2 \mathrm{H}, \mathrm{H}_{\mathrm{e}}-3, \mathrm{H}_{\mathrm{e}}-5\right), 1.89-$ $1.86\left(\mathrm{~m}, 2 \mathrm{H}, \mathrm{H}_{\mathrm{a}}-3, \mathrm{H}_{\mathrm{a}}-5\right) ;{ }^{13} \mathrm{C}-\mathrm{NMR}\left(\mathrm{CDCl}_{3}, 150\right.$ $\mathrm{MHz}, \delta(\mathrm{ppm})): 166.22$ (C-4'), 166.20 (C-2"'"'), 163.00 (C-5"'"), 157.88 (C-3"'"), 152.92 (C-6"'"'), 136.06 (C-1"'"), 134.01 (C-1"), 131.11 (C-4"), 130.33 (C-3", C-5"), 129.74 (C-2", C-6"), 128.21 (C-6"'"'), 126.99 (C-2', C-6'), 126.37 (C-1'), 123.94 (C-5"'"'), 121.87 (C-3"'"'), 117.21 (C-4"'"'), 114.21 (C-3', C-5'), 55.57 (C-1'"'), 55.23 (C-3'"'),
45.39 (C-2, C-6), 34.80 (C-2'"), 31.86 (C-4), 29.49 (C-3, C-5); EIMS (m/z): 575 [M] $]^{+}, 371,295$, 255, 171, 108, 106, 52.

Table 2: Comparison of conventional and microwaveassisted methods

\begin{tabular}{ccccc}
\hline \multirow{2}{*}{$\begin{array}{c}\text { Compo } \\
\text { und }\end{array}$} & $\begin{array}{c}\text { Reaction time } \\
\text { Conventi } \\
\text { onal (h) }\end{array}$ & $\begin{array}{c}\text { Microw } \\
\text { ave (s) }\end{array}$ & $\begin{array}{c}\text { Reaction yield (\%) } \\
\text { Conventi } \\
\text { onal }\end{array}$ & $\begin{array}{c}\text { Microw } \\
\text { ave }\end{array}$ \\
\hline 7a & 13 & 39 & 73 & 90 \\
7b & 15 & 41 & 66 & 91 \\
7c & 16 & 63 & 78 & 90 \\
$7 \mathbf{7 d}$ & 17 & 53 & 72 & 88 \\
$\mathbf{7 e}$ & 13 & 38 & 48 & 81 \\
$\mathbf{7 f}$ & 20 & 34 & 61 & 84 \\
$\mathbf{7 g}$ & 12 & 47 & 77 & 92 \\
$\mathbf{7 h}$ & 18 & 73 & 64 & 90 \\
$\mathbf{7 i}$ & 16 & 69 & 58 & 87 \\
\hline
\end{tabular}

\section{Biological activities}

The $\mathrm{IC}_{50}$ results for antioxidant and enzyme inhibition activities are given in Table 3 and the docking studies in Figures 1 to Figure 3 . The results for BSA binding studies are given in Table 4 and Figures 4 and 5.

Table 3: Antioxidant and enzyme inhibition activities

\begin{tabular}{cccc}
\hline Compound & Antioxidant & $\begin{array}{c}\mathbf{I C}_{50}(\boldsymbol{\mu M}) \\
\text { Urease }\end{array}$ & BChE \\
\hline 7a & $65.2 \pm 0.26$ & $31.1 \pm 0.29$ & $17.4 \pm 0.08$ \\
7b & $56.2 \pm 0.45$ & $45.5 \pm 0.45$ & $25.2 \pm 0.12$ \\
7c & $60.3 \pm 0.12$ & $39.5 \pm 0.65$ & $>500$ \\
7d & $49.5 \pm 0.24$ & $19.5 \pm 0.12$ & $15.5 \pm 0.77$ \\
7e & $62.2 \pm 0.45$ & $21.1 \pm 0.68$ & $22.2 \pm 0.15$ \\
$\mathbf{7 f}$ & $61.1 \pm 0.21$ & $18.2 \pm 0.78$ & $>500$ \\
$\mathbf{7 g}$ & $80.1 \pm 0.61$ & $19.9 \pm 0.77$ & $>500$ \\
$\mathbf{7 h}$ & $78.5 \pm 0.14$ & $52.4 \pm 0.60$ & $69.5 \pm 0.57$ \\
$\mathbf{7 i}$ & $76.2 \pm 0.21$ & $17.9 \pm 0.10$ & $68.2 \pm 0.22$ \\
BHA & $\mathbf{4 4 . 2 \pm 0 . 2 1}$ & & \\
Thiourea & & $\mathbf{2 4 . 3} \pm \mathbf{0 . 2 4}$ & \\
Eserine & & & $\mathbf{7 . 8} \pm \mathbf{0 . 2 4}$ \\
\hline
\end{tabular}

Table 4: Stern-Volmer quenching constants for the potent compounds warfarin and ibuprofen with BSA at 298K

\begin{tabular}{lcccc} 
Compound & $\begin{array}{c}\mathbf{K}_{\mathbf{s v} \times \mathbf{1 0}} \mathbf{1}^{\mathbf{2}} \\
\left(\mathbf{M}^{-1}\right)\end{array}$ & $\begin{array}{c}\mathbf{K}_{\mathbf{q}} \times \mathbf{1 0} \mathbf{1 0}^{\mathbf{1 0}} \\
\left(\mathbf{M}^{-1} \mathbf{s}^{-1}\right)\end{array}$ & $\begin{array}{c}\mathbf{K}_{\mathbf{a}} \\
(\mathbf{L} / \mathbf{m o l})\end{array}$ & $\mathbf{N}$ \\
\hline $7 a$ & 4.45 & 4.45 & $2.52 \times 10^{1}$ & 0.6 \\
$7 \mathrm{e}$ & 4.21 & 4.21 & $2.42 \times 10^{2}$ & 0.9 \\
Warfarin & & & 641000 & 1.07 \\
lbuprofen & 3502 & 35 & 56870 & 1.26 \\
\hline
\end{tabular}

\section{DISCUSSION}

Compound $7 \mathrm{a}$ was selected for discussion. It was obtained as a white amorphous solid with a melting point of $108.0^{\circ} \mathrm{C}$. The IR peaks at 2850 , $1700,1600,1500,1300,1250,1150$ and $750 \mathrm{~cm}$ 1 indicated different functional groups. In ${ }^{1} \mathrm{H}$ NMR, two doublet peaks appearing at 7.58 and 


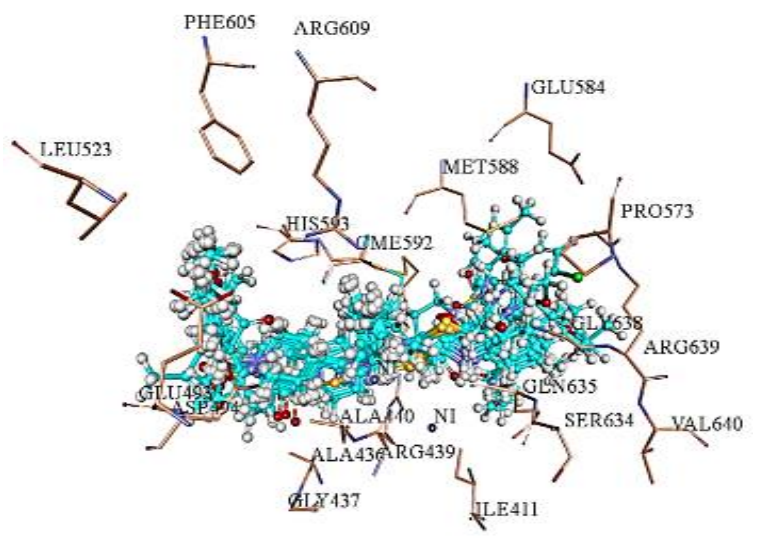

Figure 1: Binding orientation of all compounds in the active site of urease. Amino acid residues are shown in golden color and ligands in different colors

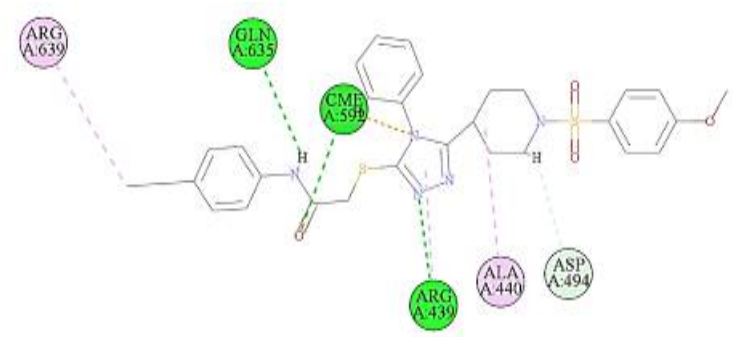

Figure 2: Binding interaction of compound $7 f$ in the active site of urease

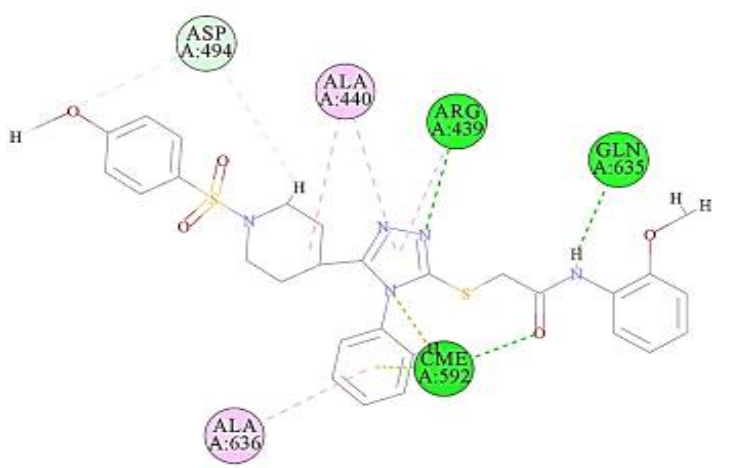

Figure 3: Binding interaction of compound $7 \mathrm{i}$ in the active site of urease

$6.99 \mathrm{ppm}$ were assigned to four protons of an aromatic ring with an attached sulfonyl group. The five protons of the phenyl ring attached to triazole were based on two multiplets at 7.567.55 and 7.26-7.21 ppm.

A doublet and two multiplets respectively appearing at 7.98, 7.26-7.21 and 7.20-7.19 ppm were assigned to the four protons of the phenyl ring attached to amidic group. The nine protons of the piperidine ring were explained by five multiplets at 3.74-3.71, 2.41-2.38, 2.35-2.33, 2.03-2.00 and 1.88-1.86 ppm. The methoxy, methylene and methyl groups were indicated by three singlets at $3.88,3.97$ and $2.31 \mathrm{ppm}$, respectively.

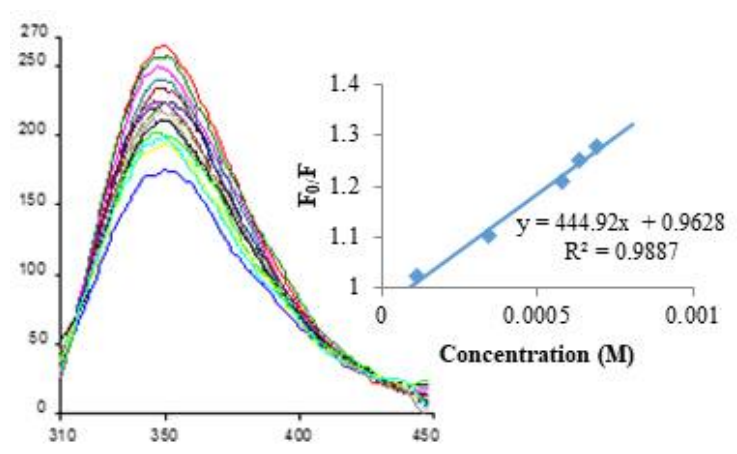

(a)

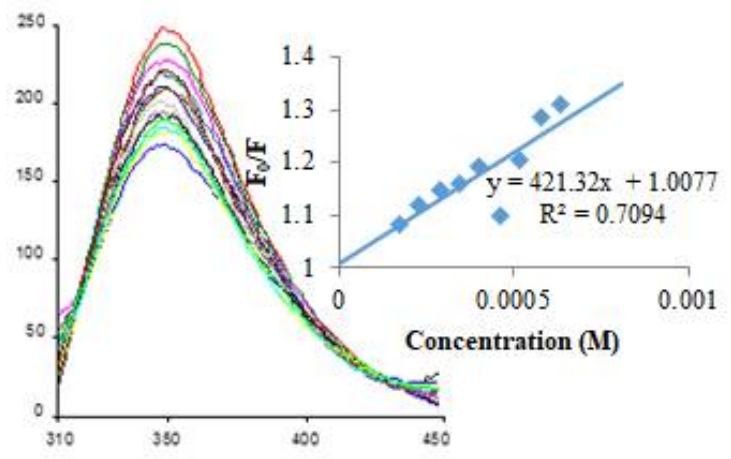

(b)

Figure 4: Fluorescence graph of BSA with (a) 7a (b) 7e. Insets show the Stern-Volmer plots.

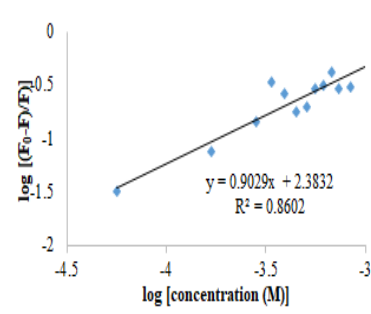

(a)

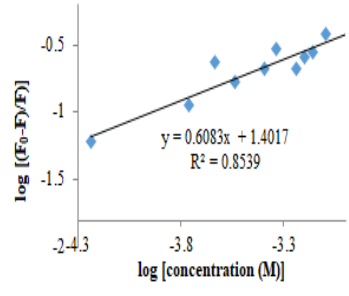

(b)
Figure 5: Fluorescence double reciprocal plots of binding of BSA with (a) $7 a$ (b) $7 e$

The aromatic and aliphatic carbons were indicated by peaks appearing at 165.58 (C-4'), 163.22 (C-5"'"'), 157.90 (C-3"'"'), 151.88 (C-6"'"'), 136.22 (C-1"'"'), 131.39 (C-1"), 130.75 (C-4"), 130.43 (C-3", C-5"), 129.64 (C-2", C-6"), 128.74 (C-2', C-6'), 127.45 (C-1' ), 126.11 (C-2'"'), 125.78 (C-6"'"'), 125.22 (C-3"'"'), 125.11 (C-5"'"'), 124.21 (C - 4"'"'), 114.20 (C-3', C-5'), 55.66 (C1"'), 45.49 (C-2, C-6), 36.34 (C-2"'), 32.01 (C-4), 29.30 (C-3, C-5 ) and 17.43 (C-3"'). The spectral data supported the identification of $7 a$ as $2-[(5-$ \{1-[(4-methoxyphenyl)sulfonyl]-4-piperidinyl\}-4phenyl-4H-1,2,4-triazol-3-yl) sulfanyl]- $N$-(2-

methylphenyl)acetamide. Although both synthetic techniques achieved high yields, the microwave- 
assisted technique was very effective in attaining high yield in less time of reaction.

Compounds 7a-7i were shown to be effective antioxidant agents, where $\mathbf{7 d}$ was the most active with an $\mathrm{IC}_{50}$ of $49.5 \pm 0.24 \mu \mathrm{M}$ compared to $44.2 \pm 0.21 \mu \mathrm{M}$ for the reference, BHA. This potential of $\mathbf{7 d}$ might have been due to 2-chloro5-methoxyphenyl as the amide aromatic ring.

All synthesized compounds displayed high urease inhibition activity as compared to the reference. Compounds $\mathbf{7 d}, \mathbf{7 e}, \mathbf{7 f}, \mathbf{7 g}$ and $\mathbf{7 i}$ were the most active with low $\mathrm{IC}_{50}$ values of 19.5 $\pm 0.12,21.1 \pm 0.68,18.2 \pm 0.78,19.9 \pm 0.77$ and $17.9 \pm 0.10 \mu \mathrm{M}$ as compared to thiourea with an $\mathrm{IC}_{50}$ of $24.3 \pm 0.24 \mu \mathrm{M}$.

BChE was inhibited by six of the nine synthesized compounds. The most active were 7a, 7b, 7d and 7e with low $\mathrm{IC}_{50}$ values. The highest potential was shown by $\mathbf{7 d}$ with the lowest $\mathrm{IC}_{50}$ value, $15.5 \pm 0.77 \mu \mathrm{M}$, which could have been due to 2-chloro-5-methoxyphenyl as the amide aromatic ring.

All the synthesized compounds were analyzed by molecular docking studies to determine the orientation in the active site of the target protein. Figure 1 shows the superimposition of all the docked compounds at the active site of urease. Binding interaction analysis of the most active compounds 7f (Figure 2) and 7i (Figure 3) indicated hydrogen bonding with Arg439 and CME592 through triazole and oxygen of acetamoyl moieties. Hydrophobic interactions with amino acid residues Ala440, Ala636 and Arg639 were also found. Hydrogen bonding is shown with green dotted line while $\pi-a l k y l$ interactions are shown with light pink dotted line.

The fluorescence emission spectral data (at an excitation of $295 \mathrm{~nm}$ ) of BSA was recorded (Table 4). The strong emission by BSA appeared at $\lambda_{\max }=336 \mathrm{~nm}$. Fluorescence decreased on binding of the synthesized compounds with BSA. The Stern-Volmer equation (Equation 1) helped to determine the nature of this fluorescence quenching, that is, static or dynamic.

$F_{0} / F=K_{S v}[Q]+1=k_{q} \tau_{0}[0]+1 \ldots \ldots(1)$

where $F$ is fluorescence intensity of BSA in the presence of compounds, $F_{0}$ is fluorescence intensity of BSA in the absence of compounds, $\mathrm{K}_{\mathrm{q}}$ is apparent bimolecular quenching rate constant, [Q] is concentration of synthesized compounds (quencher), $\mathrm{K}_{\mathrm{SV}}$ is Stern-Volmer quenching constant, and $\mathrm{T}_{0}$ is the average life time of biomolecule without synthesized compound $\left(10^{-8} \mathrm{~s}\right)$ [20]. The quenching of BSA was found to be static as indicated by the larger values of $\mathrm{k}_{\mathrm{q}}$ than maximum scattering collision quenching rate constant $\left(2 \times 10^{10} \mathrm{M}^{-1} \mathrm{~s}^{-1}\right)$.

\section{CONCLUSION}

Microwave-assisted protocol is superior to the conventional method in terms of high yield and short time. Biological screening along with docking and BSA binding studies indicate that compounds $\mathbf{7 d}, \mathbf{7 e}, \mathbf{7 f}, \mathbf{7 g}$, and $\mathbf{7 i}$ are potent antiurease agents. Thus, the current synthesis might be a good addition in the pharmaceutical industry after further biological evaluation.

\section{DECLARATIONS}

\section{Acknowledgement}

The authors acknowledge the Higher Education Commission of Pakistan for financial support.

\section{Conflict of interest}

No conflict of interest is associated with this work.

\section{Contribution of authors}

We declare that this work was done by the authors named in this article and all liabilities pertaining to claims relating to the content of this article will be borne by the authors.

\section{Open Access}

This is an Open Access article that uses a funding model which does not charge readers or their institutions for access and distributed under the terms of the Creative Commons Attribution License (http://creativecommons.org/licenses/by/ 4.0) and the Budapest Open Access Initiative (http://www.budapestopenaccessinitiative.org/rea d), which permit unrestricted use, distribution, and reproduction in any medium, provided the original work is properly credited.

\section{REFERENCES}

1. Nagender $P$, Reddy GM, Kumar RN, Poornachandra $Y$, Kumar CG, Narsaiah B. Synthesis, cytotoxicity, antimicrobial and anti-biofilm activities of novel pyrazolo[3,4-b]pyridine and pyrimidine functionalized 1,2,3-triazole derivatives. Bioorg Med Chem Lett 2014; 24: 2905-2908.

2. Omar FA, Mahfouz NM, Rahman MA. Synthesis and antimicrobial activity of 2-substituted [4-(1,3,4-oxadiazol- 
2-ylmethyl)]phthalazin-1(2H)-one derivatives. Eur J Med Chem 1996; 31: 819-825.

3. Kishore K, Jha A, Samad Y, Kumar M, Shaharyar RL, Jain KJ. Design, synthesis and biological evaluation of 1,3,4-oxadiazole derivatives. Eur J Med Chem 2010; 45 : 4963-4967.

4. Musad EA, Mohamed R, Saeed BA, Vishwanath BS, LokanathaRai KM. Synthesis and evaluation of antioxidant and antibacterial activities of new substituted bis(1,3,4-oxadiazoles), 3,5-bis(substituted)pyrazoles and isoxazoles. Bioorg Med Chem Lett 2011; 21: 3536 3540.

5. Rollas S, Gulerman N, Erdeniz IIH. Antimicrobial activity and a SAR study of some novel benzimidazole derivatives bearing hydrazone moiety. Eur J Med Chem 2002; 57: 171-174.

6. Kishore K, Jha A, Samad Y, Kumar M, Shaharyar RL, Jain KJ. Design, synthesis and biological evaluation of 1,3,4-oxadiazole derivatives. Eur J Med Chem 2010; 45 : 4963-4967.

7. Ramaprasad GC, Kalluraya B, Kumar BS, Hunnur RK. Synthesis, characterization and pharmacological activities of 2-[4-cyano-(3-trifluoromethyl)phenylamino)]4-(4-quinoline/coumarin-4-yloxy)-6-(fluoropiperazinyl)-striazines. Eur J Med Chem 2010; 45: 4587-4593.

8. Rashid M, Husain A, Mishra R. Synthesis of benzimidazoles bearing oxadiazole nucleus as anticancer agents. Eur J Med Chem 2012; 54: 855-866.

9. Kantar GK, Baltas N, Mentes E, Selami S. Microwaveassisted synthesis and investigation of xanthine oxidase inhibition of new phthalonitrile and phthalocyanines containing morpholino substituted 1,2,4-triazole-3-one. J Organomet Chem 2015; 787: 8-13.

10. Zhang $D W$, Zhang $Y$, Li J, Zhao $T$, Gu Q, Lin $F$. Ultrasonic-assisted synthesis of 1,4-disubstituted 1,2,3triazoles via various terminal acetylenes and azide and their quorum sensing inhibition. Ultrason Sonochem 2017; 36: 343-353.
11. Aziz-ur Rehman, lqbal J, Abbasi1 MA, Siddiqui $S Z$, Khalid H, Laulloo SJ, Virk NA, Rasool S, Shah SAA. Compounds with 1,3,4-oxadiazole and azinane appendages to evaluate enzymes inhibition applications supported by docking and BSA binding. Cogent Chem 2018; 4: 1441597.

12. Bondock S, Adel S, Etman HA, Badria FA. Synthesis and antitumor evaluation of some new 1,3,4-oxadiazolebased heterocycles. Eur J Med Chem 2012; 48: 192199.

13. Macaaev F, Rusu V, Pogreboni S, Gudima A, Stingaci E, Vlad I, Shvet N, Kandemirli F, Dimogloa A, Reynolds $R$. Synthesis of novel 5-aryl-2-thio-1,3,4-oxadiazoles and the study of their structure-anti-mycobacterial activities. Bioorg Med Chem 2005; 13: 4842-4850.

14. Koleva II, Van Beek TA, Linssen JPH, de Groot A, Evstatieva $L N$. Screening of plant extracts for antioxidant activity: a comparative study on three testing methods. Phytochem Anal 2002; 13: 8-17.

15. Ellman GL, Courtney KD, Andres V, Featherstone RM. A new and rapid colorimetric determination of acetyl cholinesterase activity. Biochem Pharmacol 1961; 7: 8895.

16. Mobley HL, Cortesia MJ, Rosenthal LE, Jones BD. Characterization of urease from Campylobacter pylori. $J$ Clin Microbiol 1988; 26: 831-841.

17. Elmer P. ChemBioDraw Professional Version (15.0.0.106). Cambridge Soft Waltham, MA, USA, 2017.

18. Chmara H, Andruszkiewicz R, Borowski E. Synthetic derivatives of N3-fumaroyl-L-2,3-diaminopropanoic acid inactivate glucosamine synthetase from Candida albicans. Biochim Biophys Acta 1985; 870: 357-366.

19. Valstar A, Almgren M, Brown W, Vasilescu M. The interaction of bovine serum albumin with surfactants studied by light scattering. Langmuir 2000; 16: 922-927.

20. Hu M, Wang $X$, Wang $H$, Chai $Y$, He $Y$, Song $G$. Fluorescence spectroscopic studies on the interaction of gemini surfactant 14-6-14 with bovine serum albumin. $J$ Lumin 2012; 27: 204-210. 